\title{
Determination of nonlinear elastic constants and stress monitoring in concrete by coda waves analysis
}

\author{
Cédric PAYAN ${ }^{1}$, Vincent GARNIER ${ }^{1}$, Joseph MOYSAN ${ }^{1}$ and Paul JOHNSON ${ }^{2}$ \\ ${ }^{1}$ Laboratoire de Caractérisation Non Destructive, Université de la Méditerranée, IUT \\ Aix-Provence, Avenue Gaston Berger, 13625 Aix-en-Provence Cedex 1, France \\ cedric.payan@univmed.fr \\ 2 Geophysics Group, Earth and Environmental Sciences Division, Los Alamos \\ National Laboratory of the University of California, Los Alamos, NM 875454, USA
}

\begin{abstract}
For non destructive stress evaluation in prestressed concrete, we describe in this paper a promising ultrasonic method. In "ordinary" isotropic homogeneous materials such as steel, ultrasonic determination of third order elastic constants provides means for stress evaluation since half a century. Acoustoelasticity is aimed at measuring ultrasonic velocity changes at progressively increasing applied stress. In case of concrete, example of a class of materials that exhibit strong multiple scattering as well as significant elastic nonlinear response, accurate velocity monitoring is challenging. In this paper, we purpose a novel method originally from geophysics. This treatment is aimed at detecting small changes in velocity or attenuation in the earth crust, by analyzing coda waves. So called "coda wave interferometry", this technique is transposed to concrete. We show that intense scattering can be applied to robustly determine velocity changes. CWI is implemented at progressively increasing applied stress, and thereby, we extract nonlinear elastic coefficients. We show that the nonlinear behavior of concrete is around two order magnitude greater than ordinary materials such as steel or aluminum. In addition, we show how this method could be transposed in case of in situ measurements in concrete structures and outline future possible applications.
\end{abstract}

\section{Keywords}

Nonlinear elastic parameters, coda waves, multiple scattering.

\section{Résumé}

Nous présentons dans cet article une méthode prometteuse d'évaluation de la précontrainte dans les structures en béton. Pour les milieux homogènes isotropes, les constantes élastiques $\mathrm{du}$ troisième ordre sont connues et étudiées depuis une cinquantaine d'années. L'acoustoélasticité consiste à mesurer les variations de vitesses induites par une contrainte statique. Le béton est un milieu très fortement diffusant et dont le comportement mécanique est très non linéaire. Les mesures de vitesses sont souvent obtenues avec une résolution inférieure à $1 \%$. Dans cet article, nous proposons une méthode issue de la géophysique. Elle permet de détecter de faibles variations de vitesse ou d'atténuation dans la croûte terrestre par l'analyse de la coda des signaux. Cette méthode ( "Coda Wave Interferometry »: CWI) est transposée au cas du béton. Nous montrons que la diffusion multiple peut être mise à profit pour déterminer de très faibles variations relatives de vitesses $\left(<10^{-4}\right)$. Elle est appliquée pour des niveaux de contrainte statique successivement plus élevés et permet ainsi de déterminer les constantes élastiques non linéaires. Nous montrons que le béton a une non linéarité de deux ordres de grandeurs supérieurs à celle des matériaux "ordinaires » tels l'acier ou l'aluminium. Nous montrons de plus que cette méthode peut être transposée in situ dans le cadre du suivi de l'état de santé des ouvrages d'arts en béton précontraint. 
Acoustoelastic-derived nonlinear properties of isotropic, homogeneous materials have been intensely studied for at least half a century. For instance, Hughes and Kelly [1] derived expressions for the speeds of elastic waves in a stressed solid using Murnaghan's theory of finite deformations and third-order terms in the strain-energy expression [2]. In complex materials, determining the third order constants accurately can be challenging, due to significant intrinsic dissipation, as well as heterogeneity leading to strong wave scattering. Most Earth materials fall into this class, and an extreme example is concrete. It is highly complex both chemically and mechanically, is both porous and permeable, heterogeneous and highly elastically nonlinear. In typical laboratory acoustic measurements, frequencies range from $200 \mathrm{kHz}$ to $1 \mathrm{MHz}$ in concrete. Associated wavelengths and typical aggregate sizes are equivalent, leading to strong multiple scattering into the stochastic regime. In this paper, we make use of the information carried by the waveform coda generated by multiple scattering, to obtain velocity. Applying successively larger stresses in combination with coda interferometry provides the means to evaluate velocity as a function of pressure and thereby extract the third order nonlinear coefficients.

The study of multiple scattering in the earth (termed "coda" originally by K. Aki [3]), has been of interest to the geoscience community for at least 50 years [4], for applications ranging from earthquake source localization, to location of petroleum reservoirs and to monitoring volcanic activity. The first method developed for monitoring velocity variations employing coda, termed "doublets" [referring to successive, nearly identical signals from the same Earthquake source] was proposed by Poupinet [5]. The method was refined in laboratory studies by development of the addition of monitoring changes in attenuation and applying an active source by Roberts et al. [6], where it was termed the "active doublet method". More recently developments have been aimed at detecting small changes in scattering locations due to modifications in Earth's crust, including velocity changes induced by thermal stress or stress accumulation in the crust, and source location (earthquake localization). This more recent version of the method has been broadly termed "coda wave interferometry" [7].

The purpose of this paper is to describe the development of coda wave interferometry in conjunction with incremental changes in applied stress to a specimen for determining the third order elastic constants of concrete, a method that can be applied to any solid, but is particularly appropriate for complex solids. This is accomplished by applying the coda interferometry as applied stress is step-wise increased. The essence of the method is to extract velocity and/or attenuation change between two time signals by analyzing waveform coda changes. This is accomplished by time-window, cross-correlation (intercorrelation) between signals. By inverting results from all pressure steps the Murnahagan coefficients are calculated. Recently a similar approach was applied to monitor thermally induced velocity variations in a solid [8], as well as observations of velocity changes in a sample of Berea sandstone [12]. In the following section we define the Murnaghan coefficients. We then describe details of how the coda interferometry method is implemented. This is followed by a description of the experimental procedure, analysis and results.

In the case of uniaxial loading, the strain-induced velocity variations of an initially isotropic medium can be analyzed to extract the Murnaghan third order elastic constants $l, m$, $\& n$. Considering the loading direction 1, the Murnaghan's constants are obtained by a first order approximation assuming small changes in velocities [9],

$$
l=\frac{\lambda}{1-2 v}\left[\frac{1-v}{v} L_{22}+\frac{2}{1+v}\left(L_{21}+v L_{23}\right)+2 v\right],
$$




$$
\begin{aligned}
& m=2(\lambda+\mu)\left[\frac{v}{1+v} L_{23}+\frac{1}{1+v} L_{21}+2 v-1\right], \\
& n=\frac{4 \mu}{1+v}\left[L_{21}-L_{23}-1-v\right],
\end{aligned}
$$

where $\lambda$ and $\mu$ are the second order Lame constants and $v$ is Poisson's ratio. The acoustoelastic constants $L_{i j}$ are defined as $d V_{i j} / V_{i j}{ }^{0}=L_{i j} d \varepsilon$ where $\varepsilon=d u / d x$ is the strain in the 1 direction $\left(\varepsilon_{11}\right), V_{i j}$ the speed of a wave propagating in the $i$ direction polarized in the $j$ direction and $V_{i j}{ }^{0}$ designates the wave speed in the unstrained state. We will apply these relations to extract $l, m, n$ after describing the CWI method.

In the coda interferometry method following a change in the material, velocity changes are determined by cross-correlating piecewise-in-time two waveforms, creating a sequence of the correlation functions $R^{t}(\tau)$. If no change has occurred the correlation produces a sequence of autocorrelation functions. In experiment, stress is applied sequentially stepwise in the 1 direction. The waveform corresponding stressed state is the vector $w(t)$ while $w_{0}(t)$ refers to the unstressed state. The time windowed intercorrelation function $R^{t}(\tau)$ is defined as [7]

$$
R^{t}(\tau)=\frac{\int_{t-T}^{t+T} w_{0}(t) w(t+\tau) d t}{\sqrt{\int_{t-T}^{t+T} w_{0}^{2}(t) d t \int_{t-T}^{t+T} w^{2}(t) d t}}
$$

where $2 T$ is the length of the time window and $t$ is the center position. The position of the maximum is the mean time lag $\tau$ between $w_{0}$ and $w$ in each time window (Fig. 1).

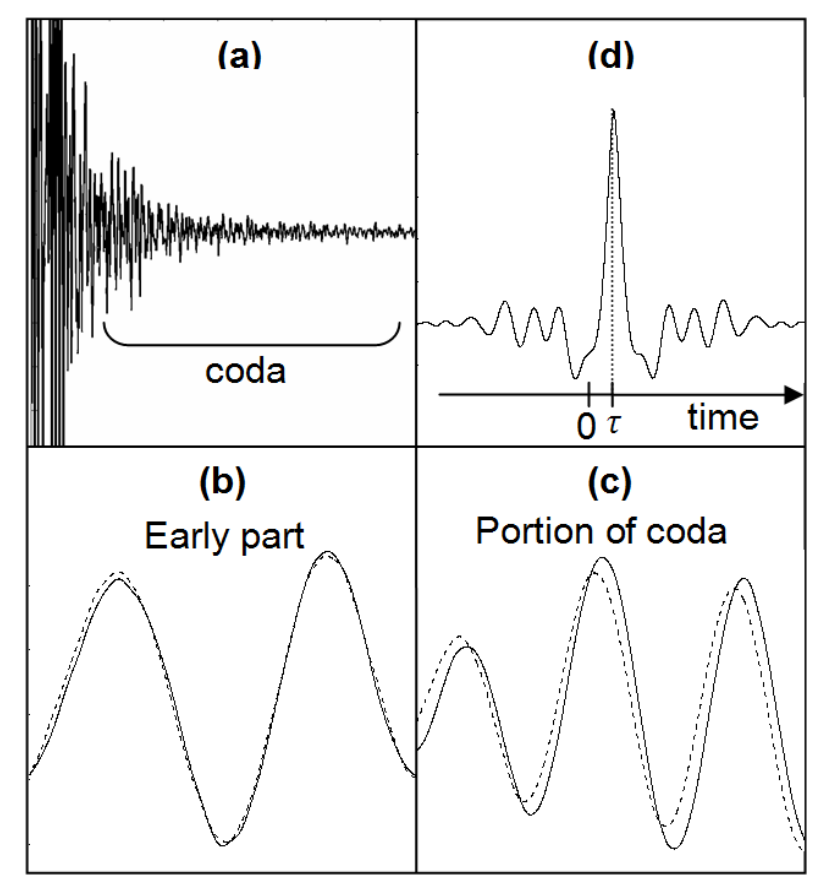

Figure 1. Analysis of coda waves. (a) Recorded signal showing coda. (b) First arrival (ballistic wave) and (c) arbitrary portion of coda taken from the portion as noted in (a).

Solid line denotes the unstressed state and dashed line the stressed state. (d) Intercorrelation function $\operatorname{Rt}(\tau)$ for extraction of the time lag $\tau$. 
Assuming the time window is small and $t>>T$, the velocity variation in each time is then obtained by [7],

$$
\frac{d V}{V^{0}}=-\frac{\tau}{t}
$$

This expression is valid for any direction considered. An advantage of this approach is that we do not require a velocity reference in the unstrained state.

Our study is performed on a cylindrical concrete sample $160 \mathrm{~mm}$ long by $75 \mathrm{~mm}$ in diameter. Strain gages were attached to the sample in order to monitor the strain in the 1 direction. Study of identical samples yielded a Young's modulus of $42.39 \mathrm{GPa}$, an ultimate strength of $76.6 \mathrm{MPa}$ and a Poisson's ratio of 0.21 . The loading protocol is determined so as to not exceed $30 \%$ of the ultimate strength in order to remain in the elastic regime [10].

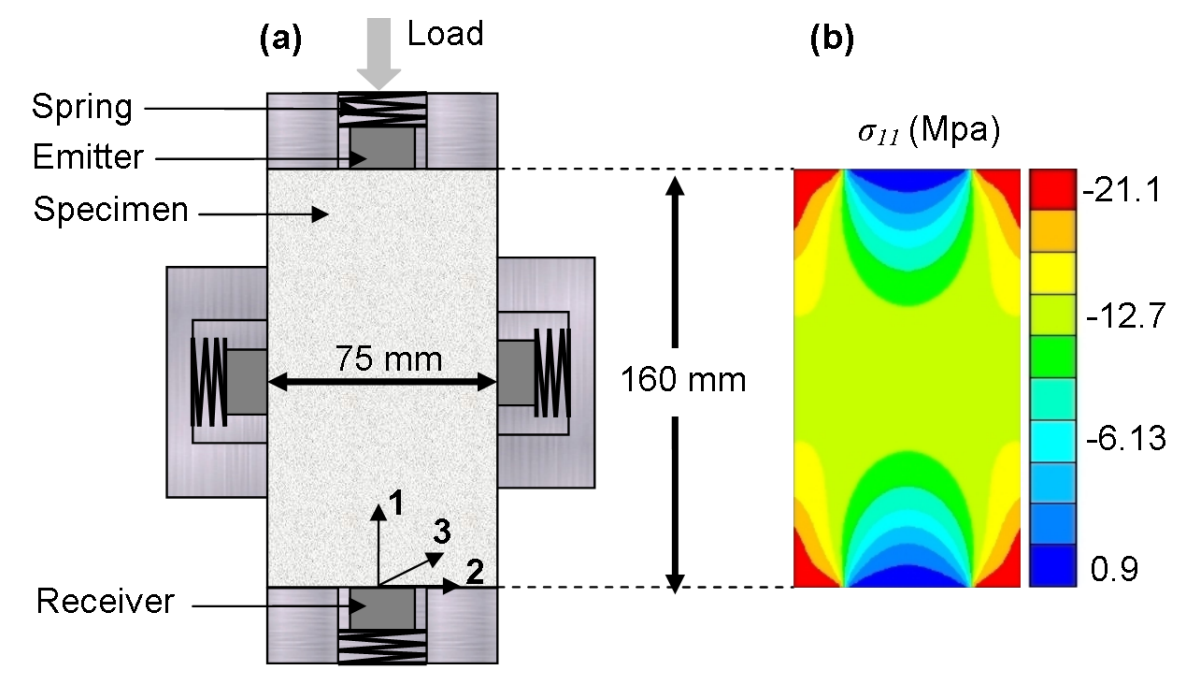

Figure 2. (a) Sample configuration. (b) Stress field $\left(\sigma_{11}\right)$ in the sample obtained by finite element analysis.

A hydraulic press (MTS 318.25) was programmed applying six stress steps from 0 to 13 $\mathrm{MPa}$. Ultrasonic transducers $25 \mathrm{~mm}$ in diameter (Sonaxis CMP36, and Panametrics V151 central frequency $500 \mathrm{kHz}$ ) were attached to the sample center at each end using ultrasonic coupling gel, and maintained in position applying constant force, using springs located in holders as shown in Fig. 2a. The transducers were driven by a high voltage system (Panametrics 5058PR). The load cell forms an annulus around the transducers (Fig. 2a). As a result the stress field in the 1 direction is not homogeneous along the 1 axis of the sample (Fig. 2b). Using $V_{21}, V_{22}, V_{23}$ in the region where stress is homogeneous (sample center) one may invert for the full set of third order elastic constants [9], by solving the linear system of equations [Eq. (1a-c)]. Measurements of $V_{11}$, and $V_{12}$ were also performed. In order to illustrate the method we present the results of the compressional wave velocity change $V_{22}$. The method is identical for the other directions.

Much empirical evidence [7,11] suggests that computing the intercorrelation function (Eq. 2 ) using 10 signal periods is optimal. In order to satisfy the assumption $t>>T$, we begin the coda analysis at $t=10 T$ ( $t=0.1 \mathrm{~ms}$ while compressional-mode time of flight is $14 \mu \mathrm{s})$ and continue to the end of the signal $(t=0.26 \mathrm{~ms})$. In this manner the relative velocity variation is computed for 8 non-overlapping windows for each stress step. Examples from the six stress steps are shown in Fig. 3.

We observe that the relative velocity variation $d V_{22} / V_{22}$ is a constant for each stress step 
but increases with stress as it should. The relative uncertainty decreases with increasing stress (from $9 \%$ to 2.3 ).

Note that the analysis is limited to $0.26 \mathrm{~ms}$. Data scatter increases in the later coda due to the fact that in late time, the high stress regions at the sample ends become important contributors (see Fig .2b).

The fits of $d V_{2 j} / V_{2 j}$ as a function of the measured strain for each applied stress for the 2 direction are given in Fig. 4. The slopes of the fits are the acoustoelastic constants $L_{i j}$ used for calculation of $l, m \& n$ from Eq. 1. The associated third order constants are shown in Table 1 . Values for granite and sandstone samples obtained from the literature are shown for comparison. As in less complex materials such as steel or aluminium, we observe that the most sensitive waves to stress are those which have particle displacement in the 1 direction, i.e. $V_{21}$ and $V_{11}$. But, in contrast to these materials, the velocity increases in all directions with stress.

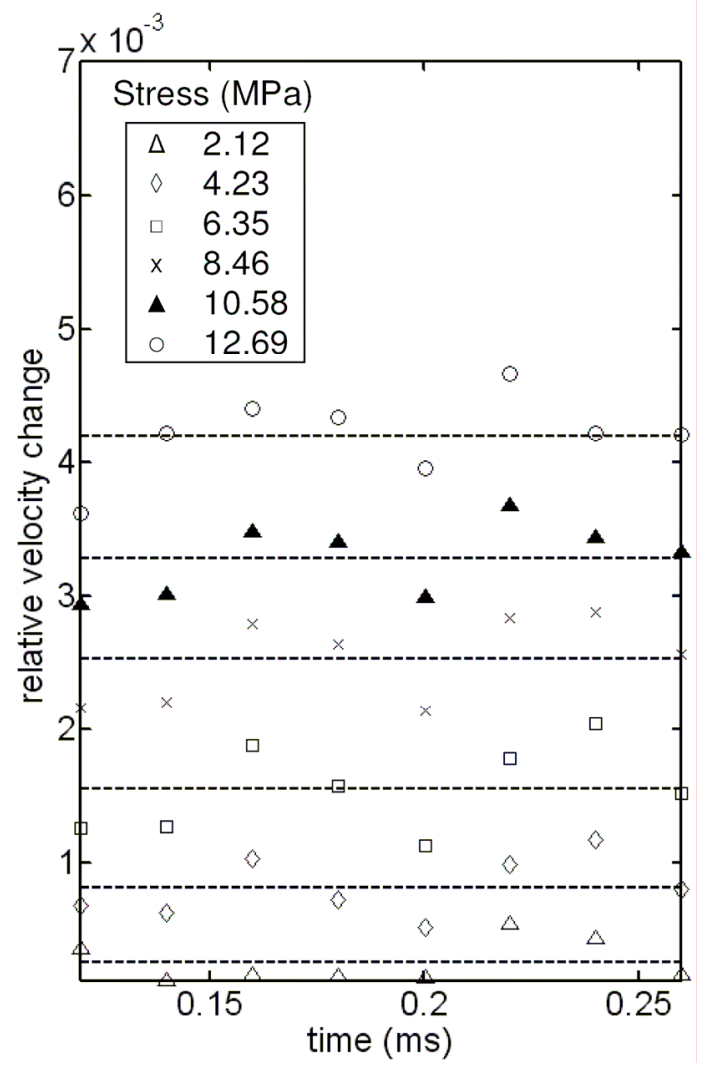

Figure 3. Result of coda wave interferometry. Dotted lines are the mean values of $d V_{22} / V_{22}$.

Table 1. Third order elastic constants and the nonlinear acoustic parameter $\beta$ in concrete, in comparison with values of two other complex materials obtained from the literature.

\begin{tabular}{|c|c|c|c|c|}
\hline & $l(\mathrm{GPa})$ & $m(\mathrm{GPa})$ & $n(\mathrm{GPa})$ & $\beta$ \\
\hline Granite [11] & $-3,371$ & $-6,742$ & $-6,600$ & -441 \\
\hline Sandstone [11] & $-97,800$ & $-99,400$ & $-84,900$ & $-9,600$ \\
\hline Concrete & $-3,007 \pm 2.8 \%$ & $-2,283 \pm 1.2 \%$ & $-1,813 \pm 3.4 \%$ & $-157 \pm 1.9 \%$ \\
\hline
\end{tabular}




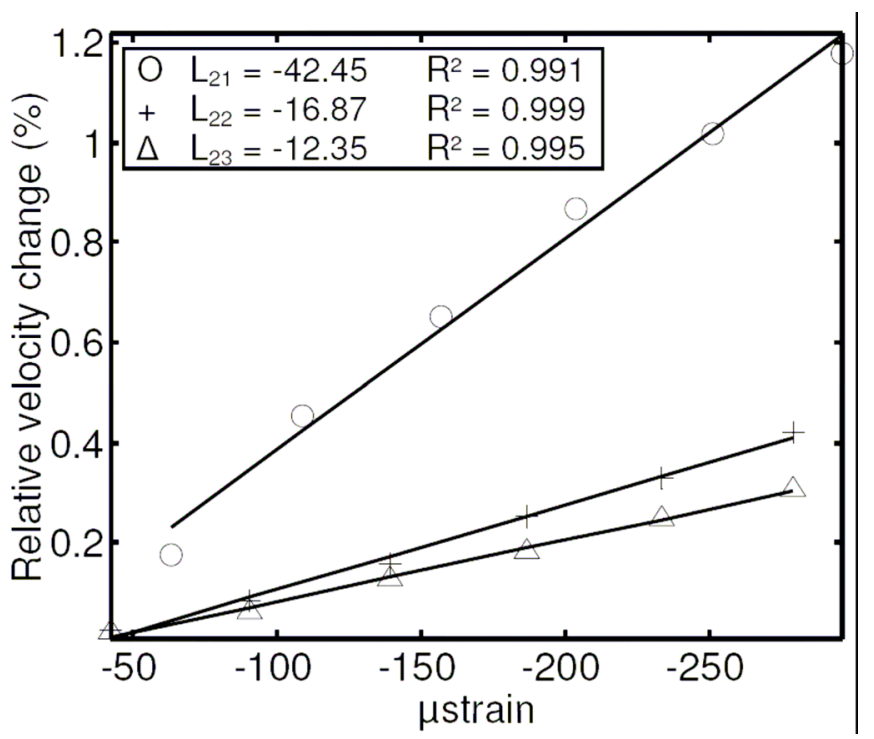

Figure 4. Relative velocity changes versus quasisatic strain used for inversion of the third order constant [see Table 1]. $R^{2}$ is the determination coefficient of the linear regression.

The local phase velocity of a sinusoidal compressional wave propagating in a onedimensional nonlinear medium can be written to first order as $V(\varepsilon)=V(0)(1+\beta \varepsilon)$, where $\beta$ is the nonlinear acoustic parameter [16]. $\beta$ can be written as a combination of Murnaghan's and Lame's elastics coefficients,

$$
\beta=\frac{3}{2}+\frac{l+2 m}{\lambda+2 \mu} .
$$

Thus the nonlinear behavior is not characterized by the absolute values of $l, m$ and $n$, but by the ratio of second and third order elastic constants. The large, negatives values are similar to those found in rock [13] and are more than two orders of magnitude greater than an ordinary nonlinear material such as steel [9]. Note the sign of $\beta$ means that as wave amplitude increases, wave velocity decreases. There are very few measurements of the third order constants of complex materials in the literature [13-15], and none to our knowledge, in concrete and cement based materials, meaning these values are the first reported for these materials.

We have presented a robust method by which to extract the third order nonlinear parameters, the Murnahgan coefficients, of concrete or any complex material. The little instrumentation needed and the simplicity of the method make possible highly accurate in situ stress monitoring measurements. In addition, by using an array of receivers, one can locate zones of stress variations.

\section{Acknowledgements}

This study was conducted in the ACTENA program supported by the French Research National Agency. We acknowledge the Laboratoire de Mécanique et d'Acoustique (Marseille in France) for technical support. PAJ was supported by the US DOE Office of Basic Energy Science. Authors thank M. Griffa for helpful comments. 


\section{References}

1. D.S. Hughes and J.L. Kelly, "Second-Order Elastic Deformation of Solids", Phys. Rev. 92, 1145 (1953).

2. F. D. Murnaghan, Finite Deformations of an Elastic Solid (John Wiley, New York, 1951).

3. K. Aki, "Correlogram analyses of seismograms by means of a simple automatic computer", J. Phys. Earth 4, 71 (1956).

4. K. Aki, "Analysis of seismic coda of local earthquakes as scattered waves", J. Geophys. Res. 74, 615 (1969).

5. G. Poupinet et al., "Monitoring Velocity Variations in the Crust Using Earthquake Doublets: An Application to the Calaveras Vault, California", J. Geophys. Res. 89, No B7, 5719 (1984).

6. P. M. Roberts, W. S. Phillips and M. C. Fehler, "Development of the Active Doublet Method for Measuring Small Velocity and Attenuation Changes in Solids", J. Acoust. Soc. Am. 91, 3291 (1992).

7. R. Snieder et al., "Coda Wave Interferometry for Estimating Nonlinear Behavior in Seismic Velocity", Science 295, 2253 (2002).

8. E. Larose et al., "Observation of multiple scattering of $\mathrm{kHz}$ vibrations in a concrete structure and application to monitoring weak changes", Phys. Rev. E 73, 016609 (2006).

9. D. M. Egle and D. E. Bray, "Measurement of acoustoelastic and 3rd-order elasticconstants for rail steel", J. Acoust. Soc. Am. 60, 741 (1976).

10. T.T.C. Hsu, "Fatigue and microcracking of concrete", Mater. Construc. 7, No 97, 51 (1984).

11. A. Grêt, R. Snieder, and U. Ozbay, "Monitoring in situ stress changes in a mining environment with coda wave interferometry", Geophys. J. Int. 167, 504 (2006).

12. A. Grêt, R. Snieder, and J. Scales, "Time-lapse monitoring of rock properties with coda wave interferometry", J. Geophys. Res. 111, B03305 (2006)

13. P. A. Johnson and P. N. J. Rasolofosaon, "Nonlinear elasticity and stress-induced anistropy in rock", J. Geophys. Res. 101, No B2, 3113 (1996).

14. P. Rasolofosaon, B. Zinszner and P. A. Johnson, "Propagation of Elastic Waves in Nonlinear Materials Survey of Laboratory Results on Rock and Geophysical Applications", Oil \& Gas Science and Technology 52, No 6, 585 (1997).

15. K. W. Winkler and X. Liu, "Measurements of third-order elastic constants in rocks", $J$. Acoust. Soc. Am. 100, 1392 (1996).

16. M. F. Hamilton, in Nonlinear Wave Propagation in Mechanics, edited by T. W. Wright (Am. Soc. Mech. Eng., New York 1986). 
NDTCE'09, Non-Destructive Testing in Civil Engineering

Nantes, France, June 30th - July 3rd, 2009 